\title{
A Study on Nature Inspired Task Scheduling Algorithms in Cloud Environment
}

\author{
N. Deepika ${ }^{1}$ and O. S. Abdul Qadir ${ }^{2}$ \\ ${ }^{1}$ Research Scholar, ${ }^{2}$ Assistant Professor \\ ${ }^{1 \& 2}$ Department of Computer Science, Jamal Mohamed College, Tiruchirappalli, Tamil Nadu, India \\ E-Mail: deepika.bca17@gmail.com
}

\begin{abstract}
Cloud computing is an encouraging paradigm which offers resources to customers on their demand with least cost. Task scheduling is the key difficult in cloud computing which decreases the performance of the system. To develop performance of the system, there is necessity of an effective task-scheduling algorithm. Nature inspired computing is a technique that is inspired by practices detected from nature. These computing techniques led to the growth of algorithms called Nature Inspired Algorithms (NIA). These algorithms are theme of computational intelligence. The persistence of raising such algorithms is to enhance engineering problems. Natureinspired algorithms have enlarged huge popularity in recent years to challenge hard real world (NP hard and NP complete) problems and resolve complex optimization functions whose actual solution doesn't occur. The paper presents a complete review of 12 nature inspired algorithms. This study offers the researchers with a single platform to analyze the conventional and contemporary nature inspired algorithms in terms of essential input parameters, their key evolutionary strategies and application areas. This study would support the research community to recognize what all algorithms could be observed for big scale global optimization to overwhelm the problem of 'curse of dimensionality'.

Keywords: Cloud Computing, Task Scheduling, Nature Inspired Algorithm
\end{abstract}

\section{INTRODUCTION}

Cloud computing is an evolving technology totally rely on internet, in which all the data and applications are hosted on a datacenters, which contains of thousands of computers interweaved together in a difficult manner. The cloud providers adopt pay as you use model for their resource use. Over the Internet, the customers can use software resources, computation power, storage space, etc., by paying money only for the period they use the resources.

Moreover Internet, customers, datacenters, and distributed servers are the central three components of a cloud eco system. Datacenter is a gathering of servers hosting diverse applications and also offers storage facility. In order to subscribe for different applications, end user wants to join tothe datacenter. Commonly a datacenter is positioned far away from the end users. Distributed servers are the portions of a cloud environment which are present through the Internet hosting different applications.The active nature of cloud computing environment wants a dynamic algorithm for efficient scheduling and load balancing between nodes. Static load balancing algorithms will works only when minor difference in the workloads. Cloud scheduling problem and load balancing problem are considered as NP hard problems. Nature inspired algorithms plays an energetic role in resolving dynamic real time problems, which are tough to resolve by regular methods. These NP hard problems are tough to solve within a time boundary. Nature inspired algorithms create optimal or near optimal solutions to these real time problems in polynomial time break. The idea behind swarm intelligence algorithm is that local contact of several simple agents to get a simple objective [1].

As the world is moving to industrialization, engineering problems are becoming more difficult and challenging to optimize. This is because of growing dimensions, time complexity, variables, space complexity etc. To cope up with such situation, nature inspired algorithms are aimed to improve numerical benchmark functions, multi objective functions and solve NP-hard problems for huge number of variables, dimensions, etc.

NIA are chiefly categorized into evolutionary algorithms and swarm intelligence based algorithms. Evolutionary algorithms are created on the evolutionary behavior of natural systems. Swarm intelligence (SI) based algorithms, also named as swarm optimization techniques; improves the definite problem by imitating the joint behavior of natural swarms' e.g. particle swarm optimization, artificial bee colony algorithm, ant colony algorithm, bacterial foraging algorithm etc. Besides these two categories, NIA have several other classifications depending on the basis of inspiratione.g. intelligent water drop algorithm is motivated from flowing of natural water drops in the river [2].

\section{RELATED WORK}

In proposed worka load balancing technique for cloud datacenter, Central Load Balancer (CLB) was suggested, which tried to evade the situation of over loading and under loading of virtual machines[3]. Based on priority and states, the Central Load Balancer accomplishes load distribution between various VMs. CLB efficiently segments the load of user requirements among several virtual machines.

Ant colony based load balancing in cloud computing was suggested in [4]. It works based on the removal of pheromone. A node with least load is fascinated by most of 
the ants. So extreme removal of pheromone happens at that node and performance is enhanced.

Cloud Light Weight (CLW) for matching the cloud computing environment workload is offered in [5]. It uses two algorithms specifically, receiver-initiated and senderinitiated approaches. VM Attribute Set is used to convince the QoS. CLW uses application migration (as the main solution) instead of using VM migration techniques in order to convince least migration time.

A resource weight based algorithm called Resource Intensity Aware Load balancing (RIAL) is projected in paper [6]. In this method, VMs are transferred from overloaded Physical Machines (PM) to casually loaded PMs. Based on resource intensity the resource weight is determined. A higher-intensive resource is given a greater weight and vice versa in each PM. The algorithm attains lower-cost and quicker convergence to the load balanced state, and reduces the probability of future load imbalance, by considering the weights when choosing VMs to migrate out and choosing destination PMs.

A cloud partitioning based load balancing model for public cloud was suggested in [7]. This algorithm applies game theory to load balancing strategy in order to increase efficiency. Here a switch mechanism is used to take dissimilar strategies for dissimilar situations.

\section{REVIEW OF NATURE INSPIRED ALGORITHMS}

The key objective of nature inspired algorithms is to discover global optimal solution for a given problem. Two key factors common in all nature inspired algorithms are intensification and diversification usually termed as Exploration and Exploitation. Exploration leads to a random search of a new solution space for finding global optima and exploitation discovers local optima in discovered solution space. Intense exploration does not offer optimal solution while deep exploitation setups an algorithm in local optima. A balance among these two factors is very necessary for any nature inspired algorithm.

TABLE I HEURISTIC AND META-HEURISTIC COMPARISON

\begin{tabular}{|l|l|l|}
\hline \multicolumn{1}{|c|}{ Type } & \multicolumn{1}{c|}{ Heuristic } & \multicolumn{1}{c|}{ Meta-Heuristic } \\
\hline Rule & $\begin{array}{l}\text { Rules are well-definedfor } \\
\text { a particularproblem } \\
\text { whichleads to a } \\
\text { SubOptimal solution. }\end{array}$ & $\begin{array}{l}\text { Rules are well-definedfor a } \\
\text { commonpurpose } \\
\text { problemwhich leads to a } \\
\text { Suboptimal solution. }\end{array}$ \\
\hline Time taken & $\begin{array}{l}\text { Provides solution in fix } \\
\text { frame of time. }\end{array}$ & $\begin{array}{l}\text { Take abundant time for } \\
\text { the solution. }\end{array}$ \\
\hline Computation & $\begin{array}{l}\text { Required } \\
\text { fewercomputation }\end{array}$ & ComputationIntensive \\
\hline Performance & $\begin{array}{l}\text { More effective } \\
\text { inperformance }\end{array}$ & $\begin{array}{l}\text { Less } \\
\text { effectiveperformance }\end{array}$ \\
\hline
\end{tabular}

Some nature inspired algorithms are discussed here[2].

A. Genetic Algorithm (GA): Genetic algorithm was specified by John Holland in 1960s and 1970s. It is a naturally motivated computational technique inspired from
Darwin's theory of evolution. It mimics the practice of natural selection. Performance of GA is based on four factors: population size, crossover rate, mutation rate and number of generations. To decide the greatest individual among population, genes of corresponding individuals are calculated against the objective function.

B. Ant Colony Optimization (ACO): Marco Dorigo proposed a new algorithm in 1992, Ant colony optimization. Ants are slight creatures that can perceptively discover the shortest path among their nest and food source. Every ant represents a possible solution to an objective function. Ants begin their communication over trails of pheromone. When an ant comes out of their nest to hunt for food source, they move casually in any direction, leaving pheromone in their path. On getting the food source, ants return back with food. Exit pheromone again on same path. Hence, the path with the top amount of pheromone denotes the shortest route from ant's nest to the food source. Pheromone concentration in every path represents the quality of solution (goodness of fitness value). The process is continued till stopping criteria is met.

C. Particle Swarm Optimization (PSO): PSO was established by J. Kennedy and R. Ederhart in 1995. PSO mimics the flocking behavior of birds. The birds fly in a solution space and their flocking behavior defines the optimum solution. They follow some path to extent their food destination. The shortest path tracked by a bird is considered to be local or particle greatest solution. Particles tend to transfer towards its local best position (solution) (lbest) found by them so far. They also keep the track of global best (gbest) solution, the best (shortest) path found by any particle at specific occurrence.The algorithm remains till global optimum solution is attained.

D. Memetic Algorithm (MA): Memetic algorithm is also motivated by Dawkin's theory of evolution. As a substitute of genes, a set memes are considered to form chromosomes. In genetic algorithm, crossover and mutation operations initiates instantly after the choice of individuals while in MA; an individual takes time to learn experience and then crossover, mutation operations are applied. When crossover among two individuals takes place, a new offspring is produced. This offspring endures local search to create local optimum solution. Individual with greatest fitness value (solution) is nominated and rests are abandoned. Thus in MA, evolutionary mechanism contains local search along with crossover and mutation.

E. Bacterial foraging optimization Algorithm (BFOA): BFOA was established by Kevin M. Passino in 2002. There are three key steps followed by bacteria to attain global optimum solution: chemotactic step, reproduction step, elimination and dispersal step. The objective of BFOA is to reduce the cost of bacteria's movement in great nutrient surface.

F. Shuffled Frog Leaping Algorithm (SFLA): SFLA was specified by Muzaffar Eusuff and Kevin Lansey in 2003. This algorithm combines the greatest properties of two 
algorithms: MA and PSO. SFLA is motivated by leaping and shuffling behavior of frogs to interchange information among them in order to hunt for food. Each frog represents a possible solution for the identified problem. After computing the fitness value of every frog in SFLA, all frogs are organized in descending order of their fitness value. Then all frogs are separated into number of subsets called memeplexes in some unusual manner; each memeplex comprises equal number of frogs. After this separation local best solution is computed within every memeplex.

G. Artificial Bee Colony Algorithm (ABC): ABC algorithm was established by Dervis Karaboga in 2005. The algorithm emulates the foraging behavior of honey bees. The term artificial bee is used because the behavior of actual bee is fairly dissimilar from the behavior of bees anticipated in $\mathrm{ABC}$ algorithm.

H. Firefly Algorithm (FFA): Firefly algorithm was first presented by Xin-She Yang in 2007. The algorithm was inspired by mimicking the flashing behavior of fireflies for the purpose food attainment. Every firefly is concerned towards other firefly. This attraction is denoted by their brightness, which rises or drops depending on distances among the flies. For all fireflies, the light intensity (brightness) of every firefly is related with other firefly.

I. Biogeography Based Optimization (BBO): $\mathrm{BBO}$ is a new bio-inspired metaheuristic algorithm suggested by Dan Simon in 2008. It is based on transferring behavior of species in habitat. Every habitat denotes a possible solution of a problem. Habitat suitability index (HSI) is an attribute of the habitat which offers the attraction of living in that habitat. Habitat immigration (species incoming in habitat) and emigration (species exit habitat) rates are decided by HSI value.

J. Cuckoo Search Algorithm (CSA): Cuckoo search algorithm is a metaheuristic algorithm established by XinShe Yang and Suash Deb in 2009. Cuckoo search algorithm (CSA) is motivated by breeding behavior of cuckoo bird. They choice their home nest by casually taking over the nest of some other birds for reproduction. They lay their eggs in particular nest of host bird and drop the host bird's egg.

K. Bat Algorithm: Bat algorithm was presented by Yang in 2010. It pretends the echolocation behavior of microbats.As microbats can make high echolocation. The Bat produces a very great sound to identify its prey which echoes back with some frequency. Echolocation is a method of discovering an object by reflected sound. It is used to recognize how far the prey is from background object.

L. Flower Pollination Algorithm (FPA): Flower pollination algorithm is a modern bio-inspired algorithm suggested by Xin-She Yang in 2012. It is motivated by fertilization (pollination) process of flowers. In FPA, abiotic and selfpollination are considered for local pollination while biotic and cross pollination is considered for the global pollination among the flower plants. The algorithm sustains a balanceamong local and global pollination.

\section{COMPARATIVE STUDY OF VARIOUS PROPOSALS}

Shiva Razzaghzadeh et al., presented a new method in order to dispense the dynamic load based on distributed queues aware of service quality in the Cloud environment [8]. In this method, they use the colorful ants as a ranking for creating distinction among the HRs capabilities. In this paper, they perform the mapping between the tasks and HRs using allocating a label to every HR. They model the load balancing and mapping process based on Poisson and exponential distribution. This model permits us to assign each task to the HR which is able to execute it with extreme power using the distributed queues aware of the service quality. Simulation outcomes display that the expert cloud can decrease the execution and tardiness time and enhance HR utilization.

Hui Wang et al., [9] presented a developed FA variant called randomly attracted FA with neighborhood search (NSRaFA). The new approach works three strategies: (1) a dynamic parameter adjustment mechanism; (2) a random attraction model; (3) three neighborhood search operators. The first strategy targets to automatically adjust the control parameters $\alpha$ and $\beta$, and evade manual parameter settings. The second one concentrates on quickening the convergence rate and decreasing the computational time complexity. The final strategy defines a new neighborhood search operation for brighter fireflies (better solutions). Searching the neighborhoods of these brighter fireflies is obliging to find well candidate solutions. Experiments are conducted on 12 famous benchmark functions to confirm the performance of their approach NSRaFA. Outcomes show that NSRaFA attains much improved solutions than the standard FA, VSSFA, WSSFA, MFA, CFA, and RaFA on the majority of test functions.

Mohit Jain et al., [10] presented a novel nature-inspired optimization paradigm, called as squirrel search algorithm (SSA). This optimizer copies the dynamic foraging behavior of southern flying squirrels and their efficient way of locomotion identified as gliding. Gliding is an effective mechanism used by small mammals for roaming lengthy distances. The work mathematically models this behavior to understand the process of optimization. The efficiency of the proposed SSA is calculated using statistical analysis, convergence rate analysis, Wilcoxon's test and ANOVA on classical as well as modern CEC 2014 benchmark functions. The proposed algorithm is applied on a real-time Heat Flow Experiment to check its applicability and robustness. The outcomes show that SSA offers more accurate solutionswith great convergence rate as related to other existing optimizers.

M. Durairaj et al., [11] suggest an approach namely Effective Cloud Resource Allocation Using Improvised Genetic Approach, which leads to achieve better virtual machine allocation across cloud servers for sustaining vertical elasticity and reducing response time. The proposed approach is focused on elasticity and scheduling 
to increase resource allocation mechanism in cloud computing. This paper not only focuses the resource utilization problem, but also converses their innovative algorithm called Enhanced Genetic Algorithm (EGA) using Multipurpose Mutation Operator. The proposed algorithm creates the effective use of mutation operator to evade local optimum problem. It repairs infeasible solutions and handles local search competently. The outcome shows that the EGA offers an optimal solution and shows better performance associated to the existing algorithms. Their method demonstrates that there is a substantial enhancement in response time and also decline in VM (Virtual Machine) migration count.

Leena V.A. et al., [12] their primary contribution is the proposal and execution of an algorithm for the simultaneous optimization of execution time and cost of scheduling, in hybrid cloud.In this work, the problem of emerging a hybrid cloud scheduler that exploits performance (or minimizes makespan) and reduces cost is addressed. This is completed by defining whether tasks have to be scheduled to either private cloud (internal cloud) or to the public cloud (external cloud). Cost can be decreased by directing more jobs to the private cloud. Execution time can be diminished by sending more jobs to public cloud. Hence both cost and execution time are contradictory objectives, as when one is made improved; other tends to be worse off. The idea is to select the best trade-off point among application completion time and cost. Heuristic algorithms can be used to attain this objective.The proposed algorithm is compared with the more broadly used scheduling optimization techniques and seen to have much improved performance.

TABle II COMPARATIVE STUdy Of VARIOUS PROPOSED WORKS

\begin{tabular}{|l|l|l|l|c|c|}
\hline \multicolumn{1}{|c|}{ Authors } & \multicolumn{1}{|c|}{ Algorithm } & \multicolumn{1}{c|}{ Concept } & \multicolumn{1}{c|}{ Objectives } & Environment & \multicolumn{1}{c|}{ Simulation } \\
\hline Shiva Razzaghzadeh et al., [8] & $\begin{array}{l}\text { Ant Colony } \\
\text { Algorithm }\end{array}$ & $\begin{array}{l}\text { Probabilistic } \\
\text { Modeling }\end{array}$ & $\begin{array}{l}\text { Improves the make span and } \\
\text { Cost }\end{array}$ & Cloud & CloudSim \\
\hline Hui Wang et al., [9] & Firefly Algorithm & $\begin{array}{l}\text { FA variant } \\
\text { (NSRaFA) }\end{array}$ & Robustness & Cloud & Cloud \\
\hline MohitJain et al.,[10] & $\begin{array}{l}\text { Squirrel Search } \\
\text { Algorithm (SSA). }\end{array}$ & $\begin{array}{l}\text { l-time Heat Flow } \\
\text { Experiment }\end{array}$ & $\begin{array}{l}\text { Checks Applicability and } \\
\text { Robustness }\end{array}$ & CloudSim \\
\hline M. Durairaj et al., & $\begin{array}{l}\text { Enhanced Genetic } \\
\text { Algorithm (EGA) }\end{array}$ & $\begin{array}{l}\text { Effective Cloud } \\
\text { Resource Allocation } \\
\text { Using Improvised GA }\end{array}$ & $\begin{array}{l}\text { Minimum make span and } \\
\text { reduces cost }\end{array}$ & CloudSim \\
\hline Leena V. A. et al., & (NSGAII Algorithm & $\begin{array}{l}\text { Bi-objective } \\
\text { optimization }\end{array}$ & $\begin{array}{l}\text { Optimization of execution time } \\
\text { and cost of scheduling in hybrid } \\
\text { cloud. }\end{array}$ & Hybrid Cloud & CloudSim \\
\hline
\end{tabular}

\section{CONCLUSION}

Nature inspired algorithms are good resolution source for real time dynamic optimization problems. Nature inspired algorithms are inspired from natural ecosystem and pretend the behavior of natural living and non-living things. This paper offered a wide review of 12 nature-inspired algorithms. The work emphasized the significant features of these algorithms in terms of their input parameters, evolutionary mechanism and applications. The key focus of this article was to clarify the research community with the optimization capability of contemporary algorithms over multi-modal and unimodal continuous functions for big scale global optimization. This paper would act as a benefit to the research community in finding the research prospects in the field of nature inspired algorithms.In future, the work will concentrate on more broad valuation of nature inspired algorithms with graphical and tabular analysis.

\section{REFERENCES}

[1] K.R. Ramesh Babu and Philip Samuel, "Enhanced Bee Colony Algorithm for Efficient Load Balancing and Scheduling in Cloud", Innovations in Bio-Inspired Computing and Applications, 2016.

[2] ParulAgarwal and Shikha Mehta, "Nature-Inspired Algorithms: Stateof-Art, Problems and Prospects", International Journal of Computer Applications, Vol. 100, August, 2014.

[3] G.D Shridharand G.R.M Reddy, "Optimal load balancing in cloud computing by efficient utilization of virtual machines”, IEEE Sixth
International Conference on Communication Systems and Networks (COMSNETS), 2014.

[4] A. Sharma and S.K. Peddoju, "Response time based load balancing in cloud computing", International Conference on Control, Instrumentation, Communication and Computational Technologies (ICCICCT), 2014.

[5] G. Soni and M. Kalra, "A novel approach for load balancing in cloud data centre", IEEE International Conference on Advance Computing Conference (IACC), 2014.

[6] S. Dam, G. Mandal, K.D asgupta, and P. Dutta, “An ant colony based load balancing strategy in cloud computing. Springer Advanced Computing”, Networking and Informatics, Vol. 28, 2014.

[7] M. Mohammadreza, M.R. Amir, and T.C. Anthony "Cloud light weight: a new solution for load balancing in cloud computing", International Conference on Data Science and Engineering (ICDSE), 2014.

[8] Shiva Razzaghzadeh, Ahmad Habibizad Navin, Amir Masoud Rahmani, and Mehdi Hosseinzadeh, "Probabilistic Modeling to Achieve Load balancing in Expert Clouds”, Ad Hoc Networks, 2017.

[9] Hui Wang, Zhihua Cui, Hui Sun, Shahryar Rahnamayan, and Xin-She Yang, "Randomly attracted firefly algorithm with neighbourhood search and dynamic parameter adjustment mechanism”, SpringerVerlag Berlin Heidelberg, 2016.

[10] Mohit Jain Vijander Singh and Asha Rani, "A novel nature- inspired algorithm for optimization: Squirrel search algorithm", Swarm and Evolutionary Computation, 2018.

[11] M. Durairaj and P. Kannan, "Improvised Genetic Approach for an Effective Resource Allocation in Cloud Infrastructure", International Journal of Computer Science and Information Technologies, Vol. 6, No. 4, 2015.

[12] V.A. Leena, A.S. Ajeena Beegom, and M.S. Rajasree, "Genetic Algorithm Based Bi-Objective Task Scheduling in Hybrid Cloud Platform”, International Journal of Computer Theory and Engineering, Vol. 8, No. 1, Feb 2016. 